\title{
A biological staging model for operable non-small cell lung cancer
}

G Cox, J L Jones, A Andi, D A Waller, K J O’Byrne

\begin{abstract}
Background-Currently the best prognostic index for operable non-small cell lung cancer (NSCLC) is the TNM staging system. Molecular biology holds the promise of predicting outcome for the individual patient and identifying novel therapeutic targets. Angiogenesis, matrix metalloproteinases (MMP)-2 and -9, and the erb/HER type I tyrosine kinase receptors are all implicated in the pathogenesis of NSCLC.

Methods-A retrospective analysis of 167 patients with resected stage I-IIIa NSCLC and $>60$ days postoperative survival with $a$ minimum follow up of 2 years was undertaken. Immunohistochemical analysis was performed on paraffin embedded sections for the microvessel marker CD34, MMP-2 and MMP-9, EGFR, and c-erbB-2 to evaluate the relationships between and impact on survival of these molecular markers.
\end{abstract}

Results-Tumour cell MMP-9 (HR 1.91 (1.23-2.97)), a high microvessel count (HR 1.97 (1.28-3.03)), and stage (stage II HR 1.44 (0.87-2.40), stage IIIa HR 2.21 (1.313.74)) were independent prognostic factors. Patients with a high microvessel count and tumour cell MMP-9 expression had a worse outcome than cases with only one (HR 1.68 (1.04-2.73)) or neither (HR 4.43 (2.29-8.57)) of these markers. EGFR expression correlated with tumour cell MMP-9 expression $(p<0.001)$. Immunoreactivity for both of these factors within the same tumour was associated with a poor prognosis (HR 2.22 (1.45-3.41)).

Conclusion-Angiogenesis, EGFR, and MMP-9 expression provide prognostic information independent of TNM stage, allowing a more accurate outcome prediction for the individual patient. The development of novel anti-angiogenic agents, EGFR targeted therapies, and MMP inhibitors suggests that target specific adjuvant treatments may become a therapeutic option in patients with resected NSCLC.

(Thorax 2001;56:561-566)

Keywords: angiogenesis; matrix metalloproteinases; non-small cell lung cancer

Dr K J O'Byrne

KOByrne@uhl.trent.nhs.uk

Received 24 August 2000

Returned to authors

18 December 2000

Revised manuscript received

14 March 2001

Accepted for publication

19 March 2001 the best prognostic index for operable NSCLC is the TNM staging system. ${ }^{2}$ However, despite potentially curative surgical resection, patients with the same pathological stage of disease display marked variability in recurrence and survival. Increasing knowledge of the molecular biology of NSCLC may allow us to predict the outcome for the individual patient. This may help to select subgroups of patients for adjuvant treatment, either with conventional cytotoxic chemotherapy or novel targeted therapeutic agents.

Angiogenesis is an important step in tumour growth and metastasis. Microvessel quantification, used as an indirect measure of angiogenesis, has been shown to be prognostic in several solid tumours including NSCLC. ${ }^{3-6}$ Matrix metalloproteinases (MMPs) are a family of endopeptidases capable of degrading basement membranes and the extracellular matrix (ECM). MMPs are frequently upregulated in malignant disease where they facilitate tumour growth, invasion, and angiogenesis. MMP-2 and MMP-9 (gelatinases A and B) are thought to be the most important MMPs in this regard. ${ }^{78}$ The erb/HER type I family of tyrosine kinase receptors is involved in cell proliferation and differentiation. Two important members of this family are EGFR and c-erbB-2 (neu/HER-2). EGFR has been shown to be associated with a poor prognosis in NSCLC in some studies ${ }^{910}$ but not in others. ${ }^{11}$ C-erbB-2 expression has also been shown to correlate with a poor outcome in some studies ${ }^{12}$ but not in all. ${ }^{10}{ }^{11}$ Recent research suggests that the erb/HER type I receptors are involved in the upregulation of MMPs. ${ }^{13} 14$

This study investigated the expression and prognostic significance of angiogenesis, MMP-2, MMP-9, EGFR, and c-erbB-2 to determine if a molecular biological staging model could be constructed.

\section{Methods}

STUDY POPULATION

A retrospective study was undertaken of 167 patients (117 men) of mean (SD) age 64.8 (7.54) years (median 66, range 42-78) with stage I-IIIa NSCLC who underwent surgical excision in Glenfield Hospital, Leicester, UK between 1991 and 1996. A preoperative staging computed tomographic (CT) scan was performed for all patients. Only enlarged mediastinal lymph nodes $(>1.5 \mathrm{~cm})$ were routinely sampled, either by preoperative mediastinoscopy or at surgical resection. Nodal clearance was not performed. The histology of each specimen was assessed according to the WHO classification $^{15}$ and the pathological stage of 
each tumour was recorded using the TNM staging system. ${ }^{2}$ Patients with a postoperative survival of $<60$ days were excluded to remove the bias of perioperative death. No patient received chemotherapy or radiotherapy prior to surgery. Follow up ranged from 24 to 85 months (median 39 months). Eighty nine subjects $(53.3 \%)$ died from a recurrence of their lung primary.

IMMUNOHISTOCHEMISTRY

The antibodies used were CD34 mouse monoclonal antibody clone QB-END/10 recognising human endothelial cells, dilution 1 in 50 (Novocastra Laboratories Ltd) ${ }^{16}$; MMP-2 mouse monoclonal antibody clone 42-5D11 recognising both latent and active MMP-2, dilution 1 in 50 (Chemicon International Ltd); MMP-9 mouse monoclonal clone 56-2A4 recognising both latent and active MMP-9, dilution 1 in 100 (Chemicon International Ltd $)^{17}$; EGFR mouse monoclonal clone EGFR.113 recognising the extracellular region of the EGFR molecule, dilution 1 in 20 (Novocastra Laboratories Ltd $)^{17}$; and HercepTest recognising the external domain of c-erbB-2 (Dako).

Formalin fixed paraffin embedded sections of tissue blocks ( $4 \mu \mathrm{m}$ thick) taken from the tumour periphery were mounted on silane coated slides. Sections were dewaxed in xylene and rehydrated through graded alcohols. Antigen retrieval for MMP-2, MMP-9, and EGFR was achieved by pressure cooking slides for 2 minutes in $10 \mathrm{mM}$ citric acid buffer at $\mathrm{pH} 6$. Endogenous peroxidase activity was blocked by treatment with $2 \%$ hydrogen peroxide for 30 minutes. Sections were rinsed in deionised water and then Tris buffered saline (TBS) containing $0.1 \%$ bovine serum albumin (BSA). To block non-specific staining slides were incubated in $20 \%$ normal rabbit serum for 10 minutes. Sections were incubated overnight at $4^{\circ} \mathrm{C}$ with the primary antibody. Sections were washed in TBS, then incubated sequentially with biotinylated rabbit anti-mouse IgG (Dako) at a dilution of 1 in 400 followed by streptavidin combined in vitro with biotinylated horseradish peroxidase at 1 in 1000 (Dako). The reaction product was developed using diaminobenzidine tetrahydrochloride. Sections were counterstained with haematoxylin, then dehydrated through graded alcohols and mounted in resinous mountant. The HercepTest protocol was followed for c-erbB-2 staining.

For each section a negative control was included where the primary antibody step was omitted and the serum was left on. In addition, a positive control preparation tissue known to contain the antigen in question was carried through with every batch of immunostain to confirm that reagents were in working order and to allow assessment of staining between batches. For many antibodies the tissue also contained an internal positive control.

MICROVESSEL QUANTIFICATION

Each section was examined under low power $(\times 40, \times 100)$ to identify the three vascular "hot spots" within the tumour. These areas were viewed at a magnification of $\times 250$ by two investigators using a 25 point Chalkley eyepiece graticule. ${ }^{318}$ We considered as a vessel any structure that stained with the chromogen, irrespective of whether a lumen was present. Only vessels within one high power field of viable tumour were deemed countable. The graticule was orientated so that the maximum number of points coincided with vessels. The total number of points from all three areas contributed to the Chalkley count. Microvessels adjacent to areas of unaffected lung parenchyma were omitted from the count. Sections were analysed by two observers in a blind fashion and the results of the clinicopathological factors and outcome were later correlated.

EVALUATION OF IMMUNOHISTOCHEMISTRY

The extent and pattern of reactivity for MMP-2, MMP-9, EGFR, and c-erbB-2 was recorded by two observers. The extent of expression was scored 0 for no staining, $<20 \%$, $20-50 \%$, and $51-100 \%$. A similar semiquantitative scale of $0,+,++$, or +++ was used to assess the intensity of staining in comparison with a known positive control. This scoring system was applied to tumour cell and stromal reactivity for MMP-2 and MMP-9 and membranous and cytoplasmic staining for EGFR. Cases were called positive if staining intensity was ++ or +++ over at least $20 \%$ of the tumour. ${ }^{17}$ The HercepTest scoring system was applied for c-erbB-2 evaluation. Sections were analysed by two observers in a blind fashion and the results of the immunohistochemistry, tumour status, and patient outcome were subsequently correlated.

ANALYSIS OF DATA

Statistical analysis was performed using the SPSS software system (SPSS for Windows Version 9.0). The $\chi^{2}$ test was used to analyse the associations between categorical clinicopathological variables. Cancer specific survival curves were plotted using the Kaplan-Meier method and the log rank test was used to assess the statistical significance of differences between groups. The joint effects of covariables that were significant at the 0.25 level in univariate analysis were further examined by Cox regression using a forward selection procedure. The 0.05 level of significance was used for entering or removing a covariable from this model.

\section{Results}

CLINICOPATHOLOGICAL FINDINGS

The clinicopathological findings are listed in table 1. Tumour spread to nodes was associated with a poor prognosis (hazard ratio (HR) 1.97 (1.30-3.00), $\mathrm{p}=0.001)$ and increasing nodal status was more significant (N1: HR 1.55 (0.96-2.52), N2: HR 3.14 (1.84-5.37), overall $\mathrm{p}=0.0001)$. The pathological stage of the tumour was prognostic (stage II: HR 1.51 (0.91-2.50), stage IIIa: HR 2.90 (1.75-4.81), overall $\mathrm{p}=0.0001$; fig $1 \mathrm{~A})$. No other clinicopathological finding including age, sex, grade, 
Table 1 Prognostic significance of tumour variables $(n=167)$

\begin{tabular}{|c|c|c|c|c|}
\hline Prognostic factor & $n$ & $\%$ & $\begin{array}{l}\text { p value } \\
\text { (log rank) }\end{array}$ & Hazard ratio \\
\hline \multicolumn{5}{|l|}{ Age (years) } \\
\hline$<$ Median & 82 & $49.1 \%$ & & 1.00 \\
\hline$\geqslant$ Median & 85 & $50.9 \%$ & 0.76 & $0.94(0.62-1.42)$ \\
\hline \multicolumn{5}{|l|}{ Sex } \\
\hline Female & 50 & $29.9 \%$ & & 1.00 \\
\hline Male & 117 & $70.1 \%$ & 0.15 & $1.41(0.88-2.25)$ \\
\hline \multicolumn{5}{|l|}{ Histology } \\
\hline Squamous & 103 & $61.7 \%$ & & 1.00 \\
\hline Adenocarcinoma & 50 & $29.9 \%$ & 0.93 & $1.01(0.64-1.60)$ \\
\hline Large cell & 14 & $8.9 \%$ & & $1.17(0.53-2.57)$ \\
\hline \multicolumn{5}{|l|}{ Grade } \\
\hline Well/moderate differentiated & 80 & $47.9 \%$ & & 1.00 \\
\hline Poorly differentiated & 87 & $52.1 \%$ & 0.18 & $1.33(0.88-2.02)$ \\
\hline \multicolumn{5}{|l|}{$\mathrm{T}$} \\
\hline 1 & 33 & $19.8 \%$ & & 1.00 \\
\hline 2 & 118 & $70.7 \%$ & 0.25 & $1.15(0.67-1.97)$ \\
\hline 3 & 16 & $9.6 \%$ & & $1.90(0.87-4.16)$ \\
\hline \multicolumn{5}{|l|}{$\mathrm{N}$} \\
\hline 0 & 88 & $52.7 \%$ & & 1.00 \\
\hline 1 & 52 & $31.3 \%$ & 0.0001 & $1.55(0.96-2.52)$ \\
\hline 2 & 27 & $16.2 \%$ & & $3.14(1.84-5.37)$ \\
\hline \multicolumn{5}{|l|}{ Stage } \\
\hline I & 84 & $50.3 \%$ & & 1.00 \\
\hline II & 47 & $28.1 \%$ & 0.0001 & $1.51(0.91-2.50)$ \\
\hline IIIa & 36 & $21.6 \%$ & & $2.90(1.75-4.81)$ \\
\hline \multicolumn{5}{|l|}{ Chalkley count } \\
\hline$>$ Median & 81 & $48.5 \%$ & 0.004 & $1.84(1.21-2.81)$ \\
\hline Upper tertile & 63 & $37.7 \%$ & 0.0002 & $2.15(1.42-3.27)$ \\
\hline \multicolumn{5}{|l|}{ MMP-2 (n=162) } \\
\hline Tumour +ve & 40 & $24.7 \%$ & 0.85 & $1.04(0.65-1.70)$ \\
\hline Stroma +ve & 100 & $61.7 \%$ & 0.15 & $0.73(0.48-1.12)$ \\
\hline \multicolumn{5}{|l|}{ MMP-9 } \\
\hline Tumour +ve & 87 & $52.1 \%$ & 0.001 & $2.02(1.31-3.10)$ \\
\hline Stroma +ve & 78 & $46.7 \%$ & 0.94 & $0.98(0.65-1.50)$ \\
\hline \multicolumn{5}{|l|}{ EGFR } \\
\hline Cytoplasm only +ve & 37 & $22.2 \%$ & 0.84 & $0.95(0.58-1.56)$ \\
\hline Membrane +ve & 55 & $32.9 \%$ & 0.14 & $1.38(0.90-2.14)$ \\
\hline Overall +ve & 92 & $55.1 \%$ & 0.24 & $1.29(0.84-1.97)$ \\
\hline Tumour cell EGFR/MMP-9 +ve & 61 & $36.5 \%$ & 0.0001 & $2.26(1.48-3.44)$ \\
\hline HercepTest score $2+$ or $3+$ & 3 & $1.8 \%$ & 0.56 & $1.50(0.37-6.10)$ \\
\hline
\end{tabular}

Hazard ratios for Chalkley count $>$ median and upper tertile are compared with counts $\leqslant$ median and lower/middle tertile counts respectively. Hazard ratios for MMP-2, MMP-9, EGFR, and HercepTest immunoexpression are compared with cases not expressing these markers.

or histological subtype was associated with outcome.

IMMUNOHISTOCHEMISTRY

Microvessel counts

Staining for CD34 was specific with minimal background staining. Normal lung tissue showed high vascularity. Interobserver variability was minimal $(R=0.78, \mathrm{p}<0.0001)$. The mean (SD) Chalkley count was 18.4 (5.30), median 18, range 8-33. A Chalkley count more than the median was associated with poor survival (HR 1.84 (1.21-2.81), $\mathrm{p}=0.004$; fig 1B), as was the upper tertile (HR 2.15 (1.42-3.27), $\mathrm{p}=0.0002)$. A Chalkley count in the upper tertile was associated with nodal disease $(p=0.02)$ and with increasing nodal spread $(\mathrm{p}=0.007)$. There was no association between Chalkley count and age, grade, or histological type.

$M M P-2$ and $M M P-9$

Reactivity for MMP-2 and MMP-9 was observed in stromal fibroblasts, infiltrating macrophages, and localised to the cytoplasm of tumour cells. MMP-2 immunostaining was seen in tumour cells of 40 of 162 cases $(24.7 \%)$ and in the stroma of 100 of 167 cases $(61.7 \%)$. Stromal expression was more commonly seen in squamous cell carcinomas $(p<0.001)$. Neither tumour cell nor stromal cell expression were associated with survival. MMP-9 tumour cell expression was recorded in 87 of 167 cases $(52.1 \%)$ and conferred a poor prognosis (HR
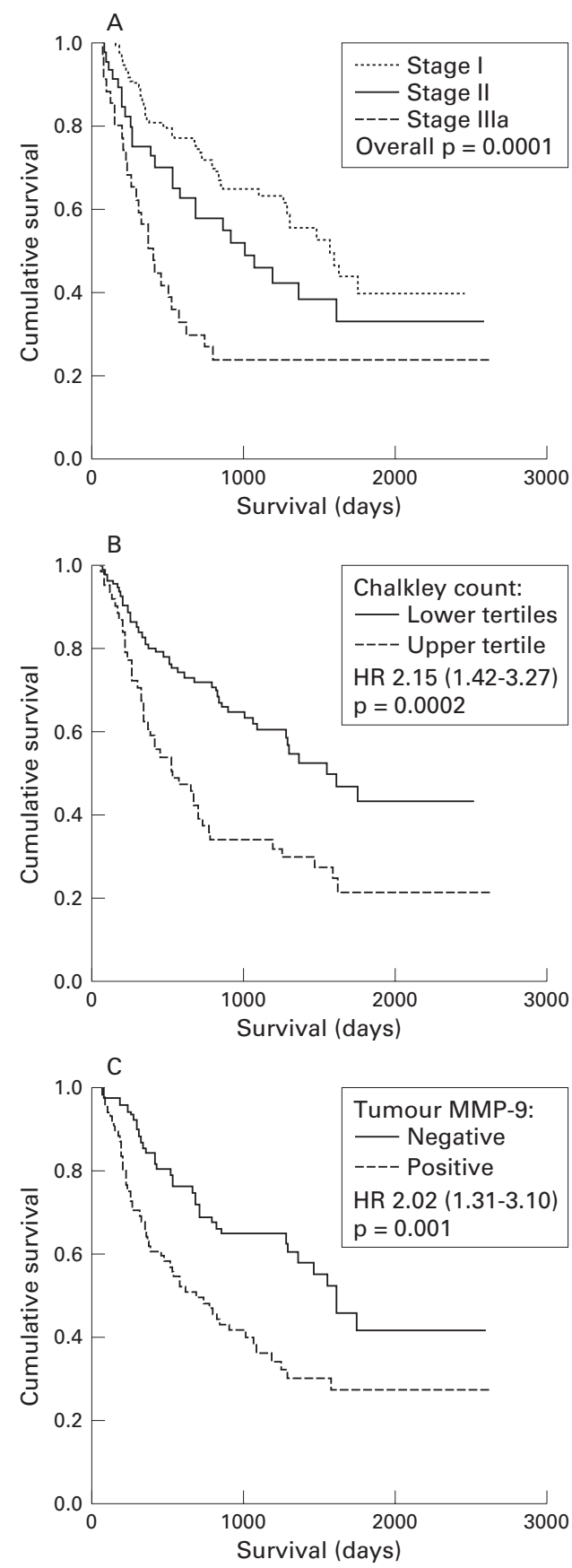

Figure 1 Kaplan-Meier survival curves for $(A)$ tumour stage, (B) Chalkley count, and (C) MMP-9 tumour cell expression.

2.02 (1.31-3.10), $p=0.001$; fig 1C). Staining was frequently more intense at the infiltrating edge of the tumour. MMP-9 stromal expression was seen in 78 of 167 cases $(46.7 \%)$ and was not prognostic. Stromal and tumour cell MMP-9 were frequently co-expressed $(p=0.009)$, and tumour cell MMP-2 and MMP-9 were frequently expressed in the same cases $(p=0.001)$. There were no associations with clinicopathological findings for tumour cell MMP-9 expression.

erb receptors

Reactivity for EGFR was present in 92 of the 167 tumours $(55.1 \%)$. Membranous immunopositivity with or without cytoplasmic staining 
Table 2 Multivariate analysis using tumour cell MMP-9 and EGFR as separate variables and as a combined variable

\begin{tabular}{lll}
\hline Factor & Hazard ratio & p value \\
\hline$M M P-9$ and EGFR as separate variables: & & \\
Chalkley count & & 0.002 \\
$\quad$ Lower tertiles & 1.00 & \\
$\quad$ Upper tertile & $1.97(1.28-3.03)$ & 0.004 \\
Tumour cell MMP-9 expression & 1.00 & \\
$\quad$ Negative & $1.91(1.23-2.97)$ & 0.01 \\
$\quad$ Positive & 1.00 & \\
Stage & $1.44(0.87-2.40)$ & \\
I & $2.21(1.31-3.74)$ & \\
II & 1.00 & \\
IIIa & $1.52(0.99-2.33)$ & \\
Grade & & \\
Well/moderately differentiated & & \\
Poorly differentiated & 1.00 & \\
MMP-9 and EGFR as combined variable: & $2.22(1.45-3.41)$ & \\
Tumour cell EGFR/MMP-9 & & \\
Negative & 1.00 & \\
Positive & $2.12(1.38-3.25)$ & \\
Chalkley count & & \\
Lower tertiles & 1.00 & \\
Upper tertile & $1.35(0.81-2.25)$ & \\
Stage & $2.29(1.36-3.84)$ & \\
I & 1.00 & \\
II & $1.31(0.86-2.01)$ & \\
IIIa & & \\
Grade & & \\
Well/moderately differentiated & & \\
Poorly differentiated & & \\
\hline & & \\
\end{tabular}

was seen in 55 (32.9\%), and cytoplasmic expression without marked membranous staining was seen in $37(22.2 \%)$. Membranous, cytoplasmic, and overall EGFR expression were not associated with outcome. Large cell and squamous cell carcinomas more frequently expressed EGFR than adenocarcinomas $(p<0.001)$. Membranous EGFR staining was associated with lower microvessel counts $(p=0.03)$. Only three of the 167 cases $(1.8 \%)$ had a HercepTest score of $2+$ or $3+$. Occasionally, single tumour cells demonstrated cytoplasmic staining.

Tumour cell expression for both MMP-9 and EGFR was found in 61 cases $(36.5 \%$; $\mathrm{p}<0.001)$, and membranous EGFR and tumour cell MMP-9 were co-expresssed in 38 cases $(22.8 \% ; \mathrm{p}=0.002)$. Expression of both EGFR and MMP-9 in the tumour cell was associated with a poor outcome (HR 2.26 (1.48-3.44), $\mathrm{p}=0.0001)$, as was expression of both membranous EGFR and MMP-9 (HR $2.04(1.28-3.26), \mathrm{p}=0.002)$.

Cox proportional hazards regression analysis was used to define biological markers with an independent predictive value with respect to cancer specific survival (table 2). Tumour cell MMP-9 or MMP-9/EGFR co-expression, high Chalkley count, and tumour stage were all significant independent prognostic factors. The addition of EGFR into the analysis increased the independent prognostic significance of the Chalkley count.

We developed a model using the molecular biological markers found to have independent prognostic significance. Patients with a high Chalkley count and tumour cell MMP-9 expression had a significantly worse prognosis than patients with only one or neither of these factors (fig 2A). Similarly, cases with both EGFR and MMP-9 tumour cell expression also produced a prognostic model (fig $2 \mathrm{~B}$ ).

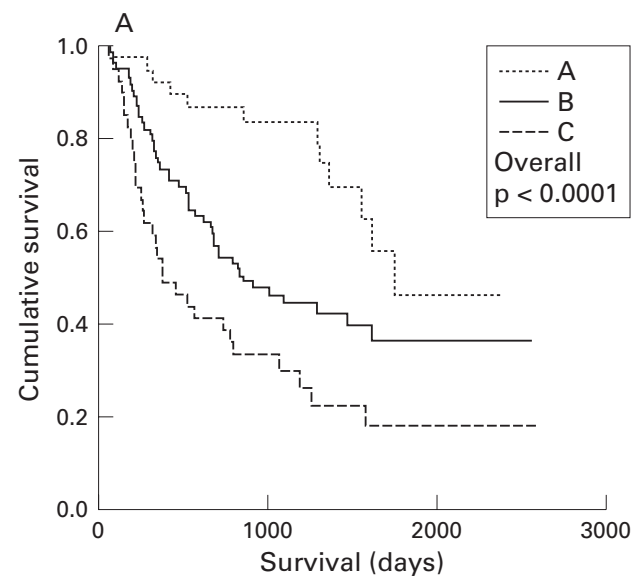

A. MMP-9 tumour negative and Chalkley count lower tertiles: 14 cancer deaths/49 (29\%)

B. MMP-9 tumour positive or Chalkley count upper tertile: 50 cancer deaths/86 (58\%)

C. MMP-9 tumour positive and Chalkley count lower tertile: 25 cancer deaths/32 (78\%)

B vs A HR 2.87 (1.58-5.20), $p=0.0003$; C vs A HR 4.43 (2.29-8.57), p < 0.0001; C vs B HR 1.68 (1.04-2.73), $p=0.03$.

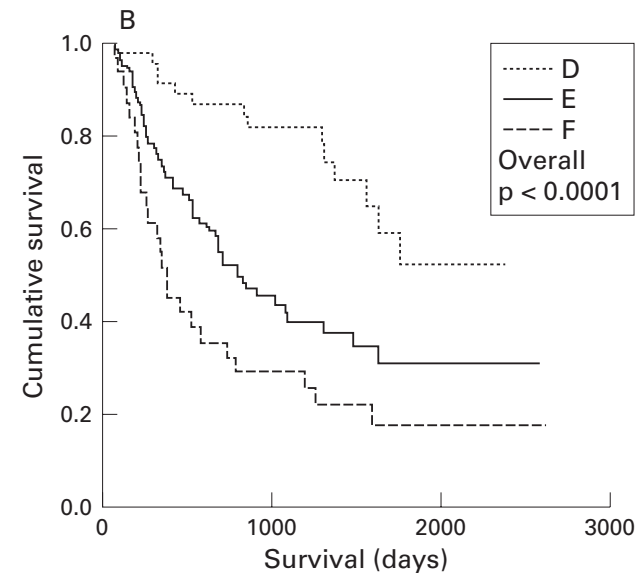

D. EGFR/MMP-9 negative and Chalkley count lower tertiles: 20 cancer deaths/63 (32\%)

E. EGFR/MMP-9 positive or Chalkley count upper tertile: 51 cancer deaths/84 (61\%)

F. EGFR/MMP-9 positive and Chalkley count lower tertile: 18 cancer deaths/20 (90\%)

E vs D HR 2.64 (1.57-4.45), $p=0.0001$;

F vs D HR 5.74 (3.00-11.0), $p<0.0001$;

F vs E HR 2.05 (1.20-3.52), $\mathrm{p}=0.01$

Figure 2 Kaplan-Meier survival curves for $(A)$ MMP-9 tumour cell expression and high Chalkley count and (B) EGFR/MMP-9 tumour cell expression and high Chalkley count.

\section{Discussion}

The goal of molecular biological staging is to find a model that will predict the long term prognosis for a patient with NSCLC on an individual basis. This will allow management decisions to be dictated by the aggressiveness of the tumour rather than the histopathological findings. Treatment may be tailored to specific metastatic pathways using novel therapeutic agents. 
The 5 year survival for stage I patients in this study was in the region of $40 \%$ (T1N0 $55 \%$, T2N0 36\%). This survival is lower than that reported in several larger studies and is probably due to surgical understaging in a proportion of the tumours.

In keeping with previous studies, microvessel density, assessed using anti-CD34 immunohistochemistry and the Chalkley counting method, was an independent prognostic factor in our patient series with high counts being associated with a poor outcome. ${ }^{3-5}$ MMP-9 tumour cell expression was significantly correlated with poor outcome, whereas stromal MMP-9 and tumour and stromal MMP-2 expression were not. Unlike MMP-2, MMP-9 is a ras-inducible gene, ${ }^{19}{ }^{20}$ which may account for the prognostic influence of MMP-9 tumour cell expression compared with that of MMP-2. Our findings, along with those from other studies, suggest that gelatinases play a significant role in NSCLC invasion and metastasis. ${ }^{21-23}$ No correlation was seen between MMP expression and microvessel density.

The relationship between upregulated EGFR expression and prognosis in NSCLC remains unclear. ${ }^{911}$ EGFR expression was not of prognostic significance in this study. Membranous EGFR expression was inversely related to angiogenesis $(p=0.03)$. This result is in keeping with a previous study in patients with NSCLC which showed erb receptor expression to be inversely related to angiogenesis and erb expressing tumours with low vascularity to have a similarly poor outcome as highly vascular tumours. ${ }^{12}$ These findings suggest that angiogenesis and the erb receptor pathways may be separate mechanisms of tumour invasion and metastasis in NSCLC.

Multivariate analysis confirmed MMP-9 tumour cell expression and a high Chalkley count to be independent molecular biological prognostic markers. To create a model for molecular sub-staging we plotted the survival curves for MMP-9 and high Chalkley count for the presence of neither, one, or both of these variables. Cases with both increased angiogenesis and MMP-9 had a significantly worse outcome than those where only one or neither of these patterns was found. Using this model, the presence of the good prognostic indices was associated with a cancer related death rate of only $29 \%$ whereas the presence of a high Chalkley count and tumour cell MMP-9 expression conferred a cancer related death rate of $78 \%$.

We found a strong correlation between EGFR immunopositivity and MMP-9 tumour cell reactivity. When a case demonstrated both EGFR and MMP-9 tumour cell expression, survival was significantly decreased. This suggests that EGFR may be involved in the specific upregulation of MMP-9, a contention supported by in vitro studies in which activation of EGFR in solid tumour cell lines resulted in upregulation of this gelatinase. ${ }^{13}{ }^{14}$ EGFR stimulation may lead to the activation of the ras/MAP kinase signal pathway and the subsequent upregulation of MMP-9. ${ }^{22}{ }^{23}$ Alternatively, EGFR may act by downregulating E-cadherin as has been seen in breast cancer. ${ }^{24}$ Downregulation of E-cadherin in mouse skin carcinoma cells results in increased MMP-9 levels associated with increased motility in vitro and metastatic potential in vivo. ${ }^{25}$ Eighteen out of 20 cases with a high Chalkley count and EGFR/MMP-9 tumour cell expression died from a recurrence of their cancer. As one of these died at day 61 with a postoperative empyema, only one out of 20 cases $(5.0 \%)$ was alive and disease free at 5 years.

This prognostic model uses only three immunohistochemical markers and would not be too time consuming to perform in a general pathology laboratory. The predictive value of this model will need to be validated by a prospective study and, if confirmed, will provide prognostic information offering the potential for individualising patient treatment. Recent work developing anti-angiogenesis agents such as angiostatin ${ }^{26}$ and endostatin, ${ }^{27}$ matrix metalloproteinase inhibitors, ${ }^{28}$ and EGFR targeted therapies ${ }^{29}$ could allow specific inhibition of these pathways, either alone or in conjunction with traditional cytotoxic treatments.

This study was financially supported by the Institute of Cancer Studies and a research grant from the Glenfield Hospital NHS Trust Research and Development Committee, Leicester.

1 Boyle P. Cancer, cigarette smoking and premature death in Europe: a review including the Recommendations of European Cancer Experts Consensus Meeting, Helsinki, Octopean Cancer Experts Consensus Meetin

2 Mountain CF. Revisions in the international system for staging lung cancer. Chest 1997;111:1711-7.

3 Fox SB, Leek RD, Weekes MP, et al. Quantification and prognostic value of breast cancer angiogenesis: comparison of microvessel density, Chalkley count and computer image analysis. F Pathol 1995;177:275-83.

4 Giatromanolaki A, Koukourakis M, O'Byrne K, et al. Prognostic value of angiogenesis in operable non-small cell lung cancer. F Pathol 1996;179:80-8

5 Fontanini $G$, Lucchi $M$, Vignati S, et al. Angiogenesis as a prospective indicator of survival in non-small-cell lung carcinoma: a prospective study. $\mathcal{F}$ Natl Cancer Inst 1997;89. $881-6$.

6 Cox G, Jones JL, Walker RA, et al. Angiogenesis and non-small cell lung cancer. Lung Cancer 2000;27:81-100.

7 Cox G, Steward WP, O'Byrne KJ. The plasmin cascade and matrix metalloproteinases in non-small cell lung cancer. Tharix metalloproteinase

8 Curran S, Murray GI. Matrix metalloproteinases in tumour invasion and metastasis. F Pathol 1999;189:300-8.

9 Veale D, Kerr N, Gibson GJ, et al. The relationship of quantitative epidermal growth factor receptor expression in non-small cell lung cancer to long term survival. Br f Cancer 1993;68:162-5.

10 Koukourakis MI, Giatromanolaki A, O’Byrne KJ, et al. Potential role of $b c l-2$ as a suppressor of tumour angiogenesis in non-small cell lung cancer. Int f Cancer 1997;74: 565-70.

11 Fontanini G, De Laurentiis M, Vignati S, et al. Evaluation of epidermal growth factor-related growth factors and receptors and of neoangiogenesis in completely resected stage I-IIIa non-small-cell lung cancer: amphiregulin and microvessel count are independent prognostic indicators in vessel count are independent progno

12 Giatromanolaki A, Koukourakis M, O’Byrne K, et al. Non small cell lung cancer: c-erbB-2 correlates with low angiogenesis and poor prognosis. Anticancer Res 1996;16:381925

13 Rosenthal EL, Johnson TM, Allen ED, et al. Role of the plasminogen activator and matrix metalloproteinase systems in epidermal growth factor- and scatter factorstimulated invasion of carcinoma cells. Cancer Res 1998;58: 5221-30.

14 O-Charoenrat $\mathrm{P}$, Rhys-Evans $\mathrm{P}$, Modjtahedi $\mathrm{H}$, et al. Overexpression of epidermal growth factor receptor in human head and neck squamous carcinoma cell lines correlates with matrix metalloproteinase-9 expression and in vitro invasion. Int $\mathcal{F}$ Cancer 2000;86:307-17.

15 World Health Organisation. The World Health Organisation histological typing of lung cancer. Am $\mathcal{f}$ Clin Pathol 1982;77:123-36.

16 Martin L, Green B, Renshaw C, et al. Examining the technique of angiogenesis assessment in invasive breast cancer. Br f Cancer 1997;76:1046-54. 
17 Cox G, Jones JL, O'Byrne KJ. MMP-9 and the epidermal growth factor receptor signal pathway in operable non-

18 Vermeulen PB, Gasparini G, Fox SB, et al. Quantification of angiogenesis in solid human tumours: an internationa consensus on the methodology and criteria of evaluation. Eur 7 Cancer 1996;32A:2474-84.

19 Tanaka H, Nishida K, Sugita K, et al. Antitumor efficacy of hypothemycin, a new Ras-signaling inhibitor. $\mathcal{F}_{p n} \mathcal{F}$ Cancer Res 1999;90:1139-45.

20 Westermarck J, Kahari V-M. Regulation of matrix metalloproteinase expression in tumor invasion. FASEB $\mathcal{F}$ 1999;13:781-92.

21 Brown PD, Bloxidge RE, Stuart NSA, et al. Association between expression of activated 72-kilodalton gelatinase and tumour spread in non-small-cell lung cancer. 7 Nat Cancer Inst 1993;85:574-8.

22 Kodate $\mathrm{M}$, Kasai $\mathrm{T}$, Hashimoto $\mathrm{H}$, et al. Expression of matrix metalloproteinase (gelatinase) in T1 adenocarcimatrix metalloproteinase (gelatinase) in

23 Suzuki M, Iizasa T, Fujisawa T, et al. Expression of matrix metalloproteinases and tissue inhibitor of matrix metalloproteinases in non-small-cell lung cancer. Invasion Metastasis 1999;18:134-41.
24 Jones JL, Royall, JE, Walker RA. E-cadherin relates to EGFR expression and lymph node metastasis in primary breast carcinoma. Br f Cancer 1996;74:1237-41.

25 Llorens A, Rodrigo I, Lopez-Barcons L, et al. Downregulation of E-cadherin in mouse skin carcinoma cells enhances a migratory and invasive phenotype linked to matrix metalloproteinase-9 gelatinase expression. $L a b$ Invest 1998;78:1131-42.

26 O'Reilly MS, Holmgren L, Shing Y, et al. Angiostatin: a novel angiogenesis inhibitor that mediates the suppression of metastases by a Lewis lung carcinoma. Cell 1994;79: 315-28.

27 O'Reilly MS, Boehm T, Shing Y, et al. Endostatin: an endogenous inhibitor of angiogenesis and tumour growth. Cell 1997;88:277-85.

28 Rasmussen HS, McCann PP. Matrix metalloproteinase inhibition as a novel anticancer strategy: a review with special focus on batimastat and marimastat. Pharmacol Ther 1997;75:69-75.

29 Ciardiello F, Bianco R, Damiano V, et al. Antitumor activity of sequential treatment with topotecan and anti-epidermal growth factor receptor monoclonal antibody C225. Clin Cancer Res 1999;5:909-16.

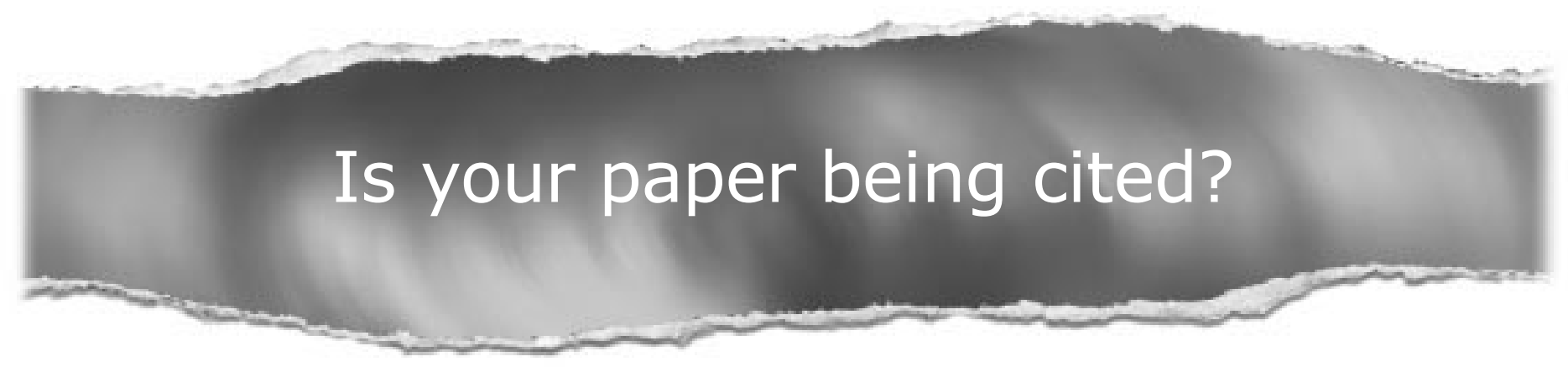

\section{CiteTrack service}

CiteTrack will alert you by email whenever new content in Thorax or a participating journal is published

that matches criteria you want to track

Topics: Tell CiteTrack which words or subjects to watch for in new content

Authors: Be alerted whenever key authors you are following publish a new paper

Articles: Know whenever a paper of interest to you is referenced by another paper

\section{www.thoraxjnl.com}

